\title{
Synthesis of Substituted Cyclopentenes from the Baylis-Hillman Adducts via Ring-Closing Metathesis Reaction
}

\author{
Ka Young Lee, Jeong Eun Na, Jin Yong Lee, ${ }^{\dagger}$ and Jae Nyoung Kim \\ Department of Chemistry and Institue of Basic Science, Chonnam National liniversity; Kiwangiu 500-757. Korea \\ 'Institute for Condensed Matter Theory and Department of Chemistry. Chonnam National University, Kwangiu 500-757, Korea \\ Received Mo: 27,2004
}

Key Words : Cyclopentenes, Baylis-I Iillman adducts, Ring-closing metathesis, Diethyl allylmalonate

Recently, we reported the synthesis of 2.5 -dihydrofuran and 2,5-dilydropyrrole systems by using the well-known ring-closing metathesis (RCM) reaction from the slightly modified Baylis-Hillman adducts. ' As a continuing effort we intended to synthesize the cyclopentene skeleton as shown in Scheme 1. Cyclopentene ring is a carbon analog of 2.5dihydrofuran and 2,5-dihydropyrrole systems and we thought we could construct the cyclopentene ring by using the similar strategy, namely the combination of BaylisHillman chemistry and RCM reaction.

Substituted cyclopentenes have been synthesized in a variety of ways ${ }^{2-1}$ including the ring-closing metathesis (RCM) reaction ${ }^{2 \cdot 1}$ and have been used as useful synthetic intermediates and act as an important backbone of some biologically important compounds.'

In order to introduce the required allyl moiety at the secondary position of the Baylis-Hillman adducts we used the well-known consecutive $\mathrm{S}_{*} 2^{\prime}-\mathrm{S}_{*} 2^{\prime}$ strategy involving DABCO salt of the bromide of the Baylis-Hillman adduct $I$, which was studied extensively by us and other groups. ${ }^{1.5}$ The reaction of dimethyl allylmalonate and the in-situ generated DABCO salt of $\mathbf{l a}$ in $\mathrm{CH}_{3} \mathrm{CN}$ gave the corresponding addition-elimination product $2 a$ in good yield $(84 \%)$. When we used aqueous THF instead of acetonitrile, the introduction of dimethyl allylmalonate recuired longer reaction time and showed lower yield of product.

With the compound $2 a$ in our hands we examined the RCM reaction. Generally the ring-closing metathesis reaction

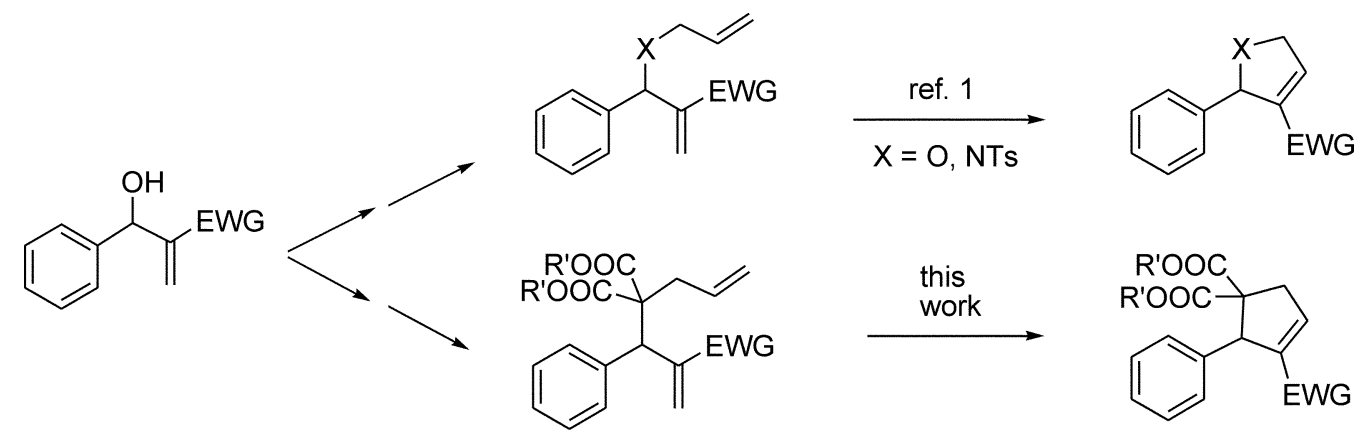

Scheme 1

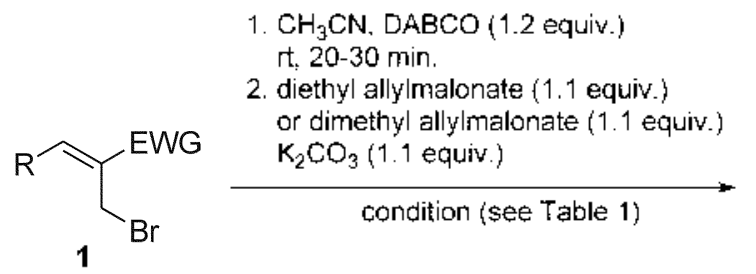

$a: R=P h, R^{\prime}=M e, E W G=C O O M e$

b: $R=P h: R^{\prime}=E t, \quad E W G=$ COOMe

c: $\mathrm{R}=\mathrm{Ph}, \mathrm{R}^{\prime}=\mathrm{Et} . \quad \mathrm{EWG}=\mathrm{COOEt}$

d: $R=P h, R^{\prime}=E t, E W G=C N$

e: $R=P h, R^{\prime}=E t, \quad E W G=$ COMe

f: $R=H, R^{\prime}=E t . \quad E W G=$ COOMe

g: $R=H, R^{\prime}=E t, \quad E W G=$ COOEt

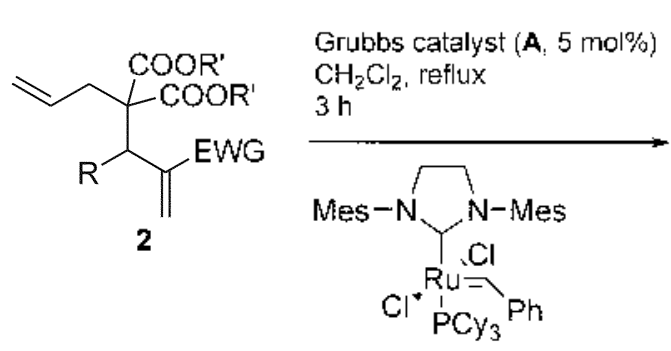

A

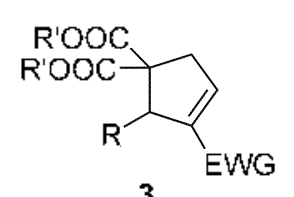

3

Sclieme 2

\footnotetext{
Corresponding Author. P'hone: -82-62-530-3381. e-mail: kimjn'tichonnam.ac.kr
} 
Table 1. Sy nthesis of dially 1 malonates 2 and cyclopentenes 3

\begin{tabular}{|c|c|c|c|}
\hline Fintry & Condition & 2 (\% Yield) & $3(\%$ Yield $)$ \\
\hline I & rt. $48 \mathrm{~h}$ & 2a (84) & 3a $(95)$ \\
\hline 2 & rt. $36 \mathrm{~h}$ & $2 b(78)$ & 3b $(89)$ \\
\hline 3 & $30-40^{\prime \prime C} .48 \mathrm{hl}$ & $2 c(80)$ & 3c (95) \\
\hline 4 & $30-40^{\circ} \mathrm{C} .44 \mathrm{hl}$ & 2d (69) & $3 d(97)$ \\
\hline 5 & $30-40^{\circ} \mathrm{C} .36 \mathrm{hl}$ & $2 \mathrm{e}(60)$ & $3 \mathbf{e}(99)$ \\
\hline 6 & $30-40^{\circ \mathrm{C}} .72 \mathrm{hl}$ & $2 \mathrm{f}(75)^{t}$ & 3f $(90)$ \\
\hline 7 & $30-40^{\circ \prime} \mathrm{C} .48 \mathrm{hl}$ & $2 \mathbf{g}(59)^{\prime}$ & $3 \mathbf{g}(93)$ \\
\hline
\end{tabular}

"The corresponding acetate was used instead of the bromide $\mathbf{I}$
$3 a$

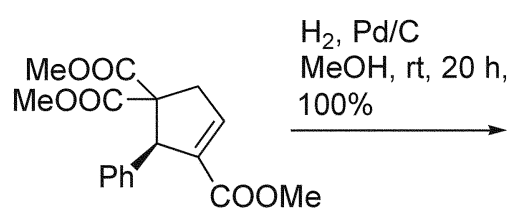

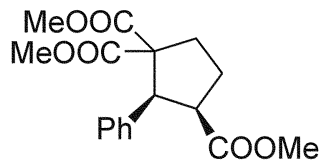

$4 a$
Scheme 3

involving electron-deficient alkene moiety as in $\mathbf{2} \mathbf{a}-\mathbf{g}$ required the use of second-generation Grubbs catalyst. ${ }^{1.6}$ The reaction of 2a under the ring-closing metathesis condition using the second-generation Grubbs catalyst (A, $5 \mathrm{~mol} \%$ ) afforded the cyclopentene derivative $\mathbf{3 a}$ in excellent yield (95\%). The representative results are summarized in lable $\mathrm{I}$. As shown, excellent yields of desired RCM products were obtained irrespective of the substituents, ester, acetyl and nitrile.

As a useful transformation, we examined the catalytic hydrogenation reaction of $\mathbf{3 a}$ using $\mathrm{Pd} / \mathrm{C}$ (Scheme 3). The reaction at room temperature ( $\mathrm{MeOH}$ under $\mathrm{H}_{2}$ balloon) produced quantitative yield of desired product $4 \mathbf{a}$ in a highly diastereoselective manner. Due to the steric hindrance of the phenyl moiety, the hydrogenation occurred at the opposite face. ${ }^{7}$ [rimethyl 2-phenylcyclopentane-1,1,3-tricarboxylate (4a) can be used for the preparation of 2-phenyl-1,3cyclopentanedicarboxylic acid ${ }^{8}$ via sequential hydrolysis and decarboxylation."

Diastereoselective decarboxylation ${ }^{9}$ and intramolecular Friedel-Crafts acylation reactions with $\mathbf{3 a - g}$ are under progress in order to prepare tricyclic compound, which could be used for further transformation.

\section{Experimental Section}

Typical procedure for the synthesis of $2 \mathrm{a}$. A mixture of the bromide la (255 $\mathrm{mg}, 1 \mathrm{mmol}$ ) and $\mathrm{DABCO}$ (135 mg, 1.2 minol) in $\mathrm{CH}_{3} \mathrm{CN}(3 \mathrm{~mL})$ was stirred at room temperature for $30 \mathrm{~min}$. To the reaction mixture $\mathrm{K}_{2} \mathrm{CO}_{3}$ (I52 $\mathrm{mg}$. I.I minol) and dimethyl allylmalonate (189 $\mathrm{mg}, 1.1 \mathrm{mmol}$ ) were added and stirred at room temperature for $48 \mathrm{~h}$. After the usual aqueous workup and column chromatographic purification process (hexanes/ether, 10:1). 2a was obtained as an oil, $291 \mathrm{mg}$ (84\%). Other compounds were synthesized similarly and their spectroscopic data are as follows.

2a: IR (neat) $1728,1631 \mathrm{~cm}^{-1} ;{ }^{1} \mathrm{H}$ NMR (CDCl $) \delta 2.63$ (dd, $J=14.1$ and $7.8 \mathrm{~Hz}, 1 \mathrm{H}$ ), 2.88 (ddt. $J=14.1,6.6$, and $1.2 \mathrm{H}$. $\mathrm{lH}$ ), $3.59(\mathrm{~s}, 3 \mathrm{H}), 3.62(\mathrm{~s}, 3 \mathrm{H}), 3.66(\mathrm{~s}, 3 \mathrm{H}), 4.79(\mathrm{~s}$, 1H), 4.99-5.02 (m, 1H), $5.06(\mathrm{~s}, 1 \mathrm{H}), 5.60-5.75(\mathrm{~m}, 1 \mathrm{H})$, $6.31(\mathrm{~d}, J=0.9 \mathrm{H} 7,1 \mathrm{H}), 6.44(\mathrm{~s}, 1 \mathrm{H}), 7.18-7.31(\mathrm{~m}, 5 \mathrm{H}) ;{ }^{13} \mathrm{C}$ NMR $\left(\mathrm{CDCl}_{3}\right) \delta 40.57,49.89,52.02,52.04,52.08,62.42$, $119.04,126.80,127.27,127.93,130.05,132.95,137.61$. $140.00,167.35,170.16,170.82$.

2b: ${ }^{1} \mathrm{H}$ NMR $\left(\mathrm{CDCl}_{3}\right) \delta 1.12(\mathrm{t}, J=7.2 \mathrm{~Hz}, 3 \mathrm{H}), 1.14(\mathrm{t} . J$ $=7.2 \mathrm{~Hz}, 3 \mathrm{H}), 2.65(\mathrm{dd}, J=14.1$ and $7.8 \mathrm{~Hz}, \mathrm{lH}), 2.90$ (ddt. $J=14.1,6.6$, and $1.2 \mathrm{~Hz}, 1 \mathrm{H}), 3.66(\mathrm{~s}, 3 \mathrm{H}), 3.99-4.15(\mathrm{~m}$, 4H), 4.80(s, 1H), 4.99-5.02 (m, 1H), 5.05-5.06(m, 1H), 5.6l$5.76(\mathrm{~m}, 1 \mathrm{H}), 6.35(\mathrm{~d}, J=0.6 \mathrm{~Hz}, 1 \mathrm{H}), 6.45(\mathrm{~s}, 1 \mathrm{H}), 7.19-7.33$ $(\mathrm{m}, 5 \mathrm{H}) ;{ }^{1.3} \mathrm{C}$ NMR $\left(\mathrm{CDCl}_{3}\right) \delta 13.73,13.84,40.50,49.64$, $52.07,61.12,61.15,62.05,118.94,126.95,127.14,127.85$, $130.19,133.06,137.75,140.04,167.43,169.73,170.39$.

2c: 'H NMR $\left(\mathrm{CDCl}_{3}\right) \delta 1.05(\mathrm{t}, J=7.2 \mathrm{~Hz}, 3 \mathrm{H}), 1.08(\mathrm{t} . J$ $=7.2 \mathrm{~Hz}, 3 \mathrm{H}), 1.11(\mathrm{t}, J=7.2 \mathrm{~Hz}, 3 \mathrm{H}), 2.58(\mathrm{dd}, J=13.8$ and $7.5 \mathrm{~Hz}, 1 \mathrm{H}), 2.84$ (ddt, $J=12,6,6.6$, and $1.2 \mathrm{~Hz}, 1 \mathrm{H}$ ), $3.92-4.09(\mathrm{~m}, 6 \mathrm{H}), 4.72(\mathrm{~s}, 1 \mathrm{H}), 4.94-5.00(\mathrm{~m}, 2 \mathrm{H}), 5.55-$ $5.69(\mathrm{~m}, 1 \mathrm{H}), 6.26(\mathrm{~s}, \mathrm{lH}), 6.38(\mathrm{~s}, 1 \mathrm{H}), 7.09-7.26(\mathrm{~m}, 5 \mathrm{H})$; ${ }^{1.9} \mathrm{C}$ NMR $\left(\mathrm{CDCl}_{3}\right) \delta 13.73,13.85,14.01,40.56,49.72$, $60.90,61.10,61.14,62.03,118.90,126.61,127.10,127.80$, $130.25,133.13,137.84,140.24,166.92,169.72,170.40$.

2d: ${ }^{1} \mathrm{H}$ NMR $\left(\mathrm{CDCl}_{3}\right) \delta 1,26(\mathrm{t}, J=7.2 \mathrm{~Hz}, 3 \mathrm{H}), 1.33(\mathrm{t} . J$ $=7.2 \mathrm{~Hz}, 3 \mathrm{H}), 2.34(\mathrm{dd}, J=14.1$ and $7.8 \mathrm{~Hz}, 1 \mathrm{H}), 2.57$ (ddt. $J=14.1,6.6$, and $1.5 \mathrm{~Hz}, 1 \mathrm{H}), 4.14-4.30(\mathrm{~m}, 3 \mathrm{H}), 4.32(\mathrm{~s}$, $1 \mathrm{H})$, 4.40-4.51 (m, 1H), 4.85-4.93 (m, 1H), 4.99-5.04 (m, 1H), 5.59-5.73 (m, 1H), $5.97(\mathrm{~s}, 1 \mathrm{H}), 6.01(\mathrm{~s}, 1 \mathrm{H}), 7.24-7.38$ $(\mathrm{m}, 5 \mathrm{H}) ;{ }^{1:} \mathrm{C}$ NMR $\left(\mathrm{CDCl}_{3}\right) \delta 13.70,14.00,39.36,53.29$, $60.84,61.69,61.81,118.48,119.36,124.15,128.23,128.82$, $129.59,132.06,134.44,135.08,169.68,169.76$.

2e: 'H NMR $\left(\mathrm{CDCl}_{3}\right) \delta 1.11(\mathrm{t}, J=7.2 \mathrm{~Hz}, 3 \mathrm{H}), 1.14(\mathrm{t}, J$ $=7.2 \mathrm{~Hz}, 3 \mathrm{H}), 2.28(\mathrm{~s}, 3 \mathrm{H}), 2.64(\mathrm{dd}, J=14.1$ and $7.5 \mathrm{~Hz}$. $1 \mathrm{H}), 2.87\left(\mathrm{ddt}_{n} J=14.1,6.9\right.$, and $\left.1.2 \mathrm{~Hz}, 1 \mathrm{H}\right), 3.95-4.17\left(\mathrm{~m}_{\mathrm{n}}\right.$ $4 \mathrm{H}), 4.92(\mathrm{~s}, \mathrm{lH}), 4.97-5.02(\mathrm{~m}, \mathrm{lH}), 5.04(\mathrm{~s}, 1 \mathrm{H}), 5.61-5.75$ $(\mathrm{m}, 1 \mathrm{H}), 6.27(\mathrm{~s}, 1 \mathrm{H}), 6.56(\mathrm{~s}, 1 \mathrm{H}), 7.17-7.32(\mathrm{~m}, 5 \mathrm{H}) ;{ }^{1 .} \mathrm{C}$ NMR $\left(\mathrm{CDCl}_{3}\right) \delta 13.74,13.87,25.91,40.50,47.69,61.07$, $61.12,62.01,118.89,126.65,127.02,127.87,130.13$, $133.12,138.19,148.63,169.89,170.45,198.75$.

2f: ${ }^{1} \mathrm{H} \mathrm{NMR}\left(\mathrm{CDCl}_{3}\right) \delta \mathrm{I} .22\left(\mathrm{t}_{.} J=7.2 \mathrm{~Hz}, 6 \mathrm{H}\right), 2.58(\mathrm{dt}, J$ $=7.2$ and $1.2 \mathrm{~Hz}, 2 \mathrm{H}), 2.95(\mathrm{~d}, J=0.9 \mathrm{~Hz}, 2 \mathrm{H}), 3.70(\mathrm{~s}, 3 \mathrm{H})$, 4.04-4.22 (m, 4H), 5.04-5.11 (m, 2H), 5.63-5.65 (m. IH), $5.66-5.76(\mathrm{~m}, \mathrm{lH}), 6.24(\mathrm{~d}, j=1.5 \mathrm{~Hz}, 1 \mathrm{H}) ;{ }^{13} \mathrm{C}$ NMR (CDCl $) \delta 13.95,33.63,37.11,51.89,57.54,61.23,119.12$. $129.11,132.47,135.91,167.38,170.53$.

2g: ${ }^{1} \mathrm{H}$ NMR $\left(\mathrm{CDCl}_{3}\right) \delta 1.25(\mathrm{t}, J=7.2 \mathrm{~Hz}, 6 \mathrm{H}), 1.29\left(\mathrm{t}_{.} J\right.$ $=7.2 \mathrm{~Hz}, 3 \mathrm{H}), 2.60$ (dt. $J=7.2$ and $1.2 \mathrm{~Hz}, 2 \mathrm{H}), 2.98(\mathrm{~d}, J=$ $0.9 \mathrm{~Hz}, 2 \mathrm{H}), 4.09-4.22(\mathrm{~m}, 6 \mathrm{H}), 5.06-5.13(\mathrm{~m}, 2 \mathrm{H}), 5.64(\mathrm{~d}, J$ $=1.5 \mathrm{~Hz}, \mathrm{lH}), 5.70-5.82(\mathrm{~m}, 1 \mathrm{H}), 6.26(\mathrm{~d}, j=1.5 \mathrm{~Hz}, 1 \mathrm{H})$; ${ }^{13} \mathrm{C} \mathrm{NMR}\left(\mathrm{CDCl}_{3}\right) \delta 13.97,14.09,33.54,37.12,57.67,60.85$, $61.23,119.05,128.75,132.58,136.24,166.95,170.55$.

Typical procedure for the ring-closing metathesis reaction of 3a. Jo a stirred solution of $\mathbf{2 a}(173 \mathrm{mg}, 0.5 \mathrm{mmol})$ in $\mathrm{CH}_{2} \mathrm{Cl}_{2}(10 \mathrm{~mL})$ was added second-generation Grubbs catalyst $(\mathbf{A}, 21 \mathrm{mg}, 5 \mathrm{~mol} \%$ ) and heated to reflux for $3 \mathrm{~h}$. After removal of solvent and column chromatographic purification process (hexanes/ether, 9:1), 3a was obtained 
as an oil. $151 \mathrm{mg}(95 \%)$. Other compounds were synthesized similarly and their spectroscopic data are as follows.

3a: IR (neat) $1736.1639 \mathrm{~cm}^{-1}:{ }^{1} \mathrm{H}$ NMR $\left(\mathrm{CDCl}_{3}\right) \delta 2.97$ (dd. $J=19.2$ and $3.0 \mathrm{~Hz}, \mathrm{IH}$ ), 3.15 (s, $3 \mathrm{H}$ ). 3.59 (s. $3 \mathrm{H}$ ). 3.67 (dt. $J=19.2$ and $2.4 \mathrm{~Hz} .1 \mathrm{H}$ ). 3.76 (s. $3 \mathrm{H}$ ). $5.04-5.06$ (m. lH). $6.87-6.90(\mathrm{~m} . \mathrm{lH}) \cdot 7 \cdot 10-7.28(\mathrm{~m}, 5 \mathrm{H}):{ }^{13} \mathrm{C} \mathrm{NMR}\left(\mathrm{CDCl}_{3}\right)$ $\delta 39.67 .51 .42 .51 .99 .53 .03,55.56,65.37,127.32,127.97$. 128.39. 137.18, 137.32, 140.41, 163.70. 168.79. 171.61: Mass ( $70 \mathrm{eV}) m z$ (rel. inensity) $59(100), 115(44), 139(38)$. 199 (47). $226(44) .258(46) .186(2 \mathrm{~L}) .318\left(\mathrm{M}^{-} .32\right)$.

3b: ${ }^{1} \mathrm{H}$ NMR $\left(\mathrm{CDCl}_{3}\right) \delta 0.86(\mathrm{t}, J=7.2 \mathrm{~Hz}, 3 \mathrm{H}), 1.25(\mathrm{t}, J$ $=7.2 \mathrm{~Hz}, 3 \mathrm{H}), 2.96($ dd $J=19.5$ and $3.0 \mathrm{~Hz}, 1 \mathrm{H}), 3.46-3.52$ (m. $\mathrm{lH}) .3 .59(\mathrm{~s}, 3 \mathrm{H}) .3 .65-3.75(\mathrm{~m}, 2 \mathrm{H}) .4 .15-4.3 \mathrm{l}(\mathrm{m} .2 \mathrm{H})$. 5.03-5.05 (m. lH), 6.86-6.89 (m, lH), 7.12-7.27 (m, 5H): ${ }^{13} \mathrm{C}$ NMR $\left(\mathrm{CDCl}_{\mathfrak{j}}\right) \delta 13.43,13.93,39.88 .51 .48,55.43$. $61.31,61.89,65.25,127.34 .128 .00$. 128.65, 137.54, 137.63. $140.35 .163 .83,168.55 .171 .25$

3c: ${ }^{1} \mathrm{H} \mathrm{NMR}\left(\mathrm{CDCl}_{\hat{j}}\right) \delta 0.77(\mathrm{t}, J=7.2 \mathrm{~Hz} .3 \mathrm{H}) .1 .01(\mathrm{t}, J=$ $7.2 \mathrm{~Hz}, 3 \mathrm{H}$ ), $1.17(\mathrm{t}, J=7.2 \mathrm{~Hz}, 3 \mathrm{H}), 2.87$ (dd, $J=19.5$ and $3.0 \mathrm{~Hz} . \mathrm{lH}) .3 .37-3.44(\mathrm{~m}, \mathrm{lH}), 3.58-3.67$ (m. $2 \mathrm{H}) .3 .87-4.05$ (m. 2H). 4.10-4.75 (m. 2H). 4.96-4.98 (m. IH). 6.78-6.80 (m. liH). 7.05-7.15 (m, 5H); ${ }^{13} \mathrm{C} \mathrm{NMR}\left(\mathrm{CDCl}_{3}\right) \delta 13.28 .13 .75$. 13.79, 39.82, 55.44. 60.05.61.13.61.72.65.07. 127.10. 127.79, $128.58 .137 .78,137.82 .139 .81,163.23,168.46 .171 .16$.

3d: ${ }^{1} \mathrm{H}$ NMR $\left(\mathrm{CDCl}_{j}\right) \delta 0.81(\mathrm{t}, J=7.2 \mathrm{~Hz}, 3 \mathrm{H}), 1.26(\mathrm{t}, J$ $=7.2 \mathrm{~Hz} .3 \mathrm{H}), 2.93-3.01(\mathrm{~m} . \mathrm{lH}) .3 .39-3.45(\mathrm{~m}, 1 \mathrm{H}), 3.65-$ $3.74(\mathrm{~m} .2 \mathrm{H}), 4.17-4.34(\mathrm{~m} .2 \mathrm{H}) .5 .05(\mathrm{~s}, \mathrm{lH}), 6.73-6.76(\mathrm{~m}$. lH), 7.14-7.31 (m, 5H): ${ }^{13} \mathrm{C}$ NMR $\left(\mathrm{CDCl}_{3}\right) \delta 13.35,13.93$. $40.73,57.53,61.69,62.25,64.54$. 115.07, 117.00, 128.31 . $128.45 .128 .93,135.23 .145 .53 .168 .06,170.54$.

3e: ${ }^{1} \mathrm{H} \mathrm{NMR}\left(\mathrm{CDCl}_{3}\right) \delta 0.80$ (t. $\left.J=7.2 \mathrm{~Hz}, 3 \mathrm{H}\right) .1 .18$ (t. $J$ $=7.2 \mathrm{~Hz} .3 \mathrm{H}) .2 .1+(\mathrm{s}, 3 \mathrm{H}), 2.94(\mathrm{dd} . J=19.5$ and $3.0 \mathrm{~Hz}$. $1 \mathrm{H}), 3.36-3.47(\mathrm{~m}, \mathrm{lH}), 3.60-3.7 \mathrm{l}(\mathrm{m}, 2 \mathrm{H}), 4.05-4.25(\mathrm{~m}$, $2 \mathrm{H}), 5.00(\mathrm{~s} . \mathrm{HH}) .6 .71-6.74$ (m. IH), 7.02-7.20 (m. $5 \mathrm{H}):{ }^{13} \mathrm{C}$ NMR $\left(\mathrm{CDCl}_{3}\right) \delta 13.44 .13 .94 .27 .11,40.24,55.13,61.34$. 61.89, 65.12. 127.31. 128.05, 128.61, 137.74. 139.86. 146.23. 168.63, 171.27. 194.47.

3f: ${ }^{1} \mathrm{H} \mathrm{NMR}\left(\mathrm{CDCl}_{3}\right) \delta 1.19(\mathrm{t}, J=7.2 \mathrm{~Hz}, 6 \mathrm{H}), 3.09-3.12$ (m. 2H). $3.17-3.20$ (m. 2H). 3.67 (s. $3 \mathrm{H}$ ). 4.13 (q. $J=7.2 \mathrm{~Hz}$. $4 \mathrm{H}) .6 .55$ (quintet. $J=2.1 \mathrm{~Hz}, 1 \mathrm{H}) .{ }^{13} \mathrm{C} \mathrm{NMR}\left(\mathrm{CDCl}_{3}\right) \delta 13.92$. 39.40, 41.01, 51.52, 58.67, 61.78, 133.41, 139.72, 164.41. 171.27 .

$3 \mathrm{~g}$ : ${ }^{\circ} \mathrm{H} \mathrm{NMR}\left(\mathrm{CDCl}_{3}\right) \delta 1.26$ (t. $\left.J=7.2 \mathrm{~Hz}, 6 \mathrm{H}\right), 1.29$ (t. $J$ $=7.2 \mathrm{~Hz} .3 \mathrm{H}) .3 .16-3.19(\mathrm{~m} .2 \mathrm{H}) \cdot 3.24-3.27(\mathrm{~m} .2 \mathrm{H}) .4 .19$ (q. $J=7.2 \mathrm{~Hz}, 2 \mathrm{H}$ ) 4.21 (q. $J=7.2 \mathrm{~Hz}, 4 \mathrm{H}$ ), 6.61 (quintet. $J$ $=2.4 \mathrm{~Hz}, 1 \mathrm{H}):{ }^{13} \mathrm{C} \mathrm{NMR}\left(\mathrm{CDCl}_{3}\right) \delta 13.90,14.13,39.38$. $40.97,58.63,60.33,61.73,133.74,139.33,163.96,171.29$.

Catalytic hydrogenation of 3a. A mixture of $3 \mathrm{a}(200 \mathrm{mg}$. $0.63 \mathrm{mmol})$ and $10 \% \mathrm{Pd} / \mathrm{C}(28 \mathrm{mg})$ in methanol $(3 \mathrm{~mL})$ was stirred at room temperature for $20 \mathrm{~h}$ under $\mathrm{H}_{2}$ atmosphere (using balloon). After removal of solvent and column chromatographic purification (hexanes/ether. $2: 1$ ) we obtained 4a (201 mg. oil) quantitatively: ' $\mathrm{H}$ NMR $\left(\mathrm{CDCl}_{3}\right) \delta 1.86-$ $2.01(\mathrm{~m} . \mathrm{lH}) .2 .21-2.37(\mathrm{~m} .2 \mathrm{H}) .2 .69-2.80(\mathrm{~m} .1 \mathrm{H}) .3 .16$ (s. $3 \mathrm{H}) .3 .28-3.38$ (m. $1 \mathrm{H}) .3 .58$ (s. $3 \mathrm{H}) .3 .74$ (s. $3 \mathrm{H}) .4 .36$ (d. $J$ $=10.8 \mathrm{~Hz}, 1 \mathrm{H}) .7 .16-7.29(\mathrm{~m} .5 \mathrm{H}):{ }^{13} \mathrm{C}$ NMR $\left(\mathrm{CDCl}_{3}\right) \delta$ $28.89,34.44 .49 .62,52.08,52.19 .52 .97,54.12,65.63$.
127.47, 128.26. 128.81, 138.57, 171.05. 172.41, 174.43.

Acknowledgments. This work was supported by the grant (R05-2003-000-10042-0) from the Basic Research Program of the Korea Science \& Engineering Foundation. Spectroscopic data was obtained from the Korea Basic Science Institute. Kwangju branch.

\section{References and Notes}

1. Kiml. T. M.: Lee. K. Y.: Lee. S.-K.: Kim. J. N. Tetrahedhon Lett. 2004. 45,2805 .

2. For the synthesis of cyclopentene derivatives by ring-closing metathesis reaction. see: (a) Grela. K.: Kim. M. Ew: J. Org. Chem. 2003. 963. (b) Eqans. P. A.: Kennedy. L. J. Tetrhedron Lett. 2001. 42. 7015. (c) Wakamatsu. H: Blechert. S. Angew: Chem. Int. Ed. 2002. 794. (d) Nugent. W. A.: Feldman. T.: Calabrese. J. C. J. Am. Chent. Soc. 1995. 117,8992, (e) Fu, G. C.: Nguyen. S. T.; Grubbs. R. H. J. Am. Chem. Soc. 1993, 115.9856. (f) Coates. G. W. Grubbs, R. H. J. Am. Chom. Soc. 1996. 118, 229. (g) Alexander, J. B.: La, D S.: Cefäo. D. R.: Hoveyda. A. H.: Schrock. R. R. J. Am. Chem. Soc. 1998. 120. 4041. (h) Furstner. A.: Thiel. O. R.: Ackermann. L: Schatz. H.-J.: Nolan. S. P. J. Ohg Chem. 2000. 65. 2204. (i) Chao. W. Weinreb, S. M. Org. Lett. 2003. 5, 2505. (j) Yang, C.: Murray, W. V: Wilson. L. J. Tetrahedon Lett. 2003. 44. 1783.

3. For the synthesis of cyclopentene ring by other methods, see: (a) Du. Y.: Lu. X: Zhang. C. Angew: Chem. Int Ed 2003. 42.1035. (b) Zhu. G.: Chen. Z: Jiang. Q: Xiao. D.: Cao. P:: Zhang. X. J. Ant Chent. Soc. 1997. 119. 3836. (c) Zhang. C.: Lu. X. J. Org Chent 1995. 60. 2906. (d) Allan, R. D.: Duke, R. K. Hambley, T. W.: Johnston. G. A. R.: Mewett, K. N.: Quickert. N.: Tran, H. W. Atust J. Chem 1996. 49. 785. (e) Citterio. A.: Sebastiano, R: Nicolini. M. Tendhedron 1993. 49.7743. (t) Sakito. Y: Suzukamo. G. Chem. Lett. 1986. 621. (g) Stevens. H. C.: Rinehart. J. K: Latanish. J. M.: Trenta. G. M. J. Ong. Chent. 1971. 36. 2780.

4. For the synthesis of biologically important compounds containing cyclopentene sheleton. see: (a) Thorstensson, F.: Kvarnstrom, I: Musil. D.: Nilsson, I.: Samuelsson, B. J. Hed Chem. 2003. to, 1165. (b) Holt. D. T.: Barker. W. D.: Jenkins. P. R. J. Org. Chem. 2000. 65. 482. (c) Fang. Z.: Hong. J. H. Org. Lett. 2004. 6.993. (d) Hale. K. I: Domostoj. M. M.: Tocher. D. A.: Irving. E: Scheinmann. F. Ong. Lett. 2003, 5, 2927. (e) Chavez. D. E.: Jacobsen, E. N. Org. Lett. 2003. 5. 2563. (f) Seepersuld, M:: AlAbed, Y. Org. Lett 1999, 1, 1463. (g) Gillaizeau, I.; Charamon, S.: Agrotoglio. L. A. Tetrahadron Lett. 2001. 42.8817. (h) Handa. S.: Earlam. G. I.: Geary. P. J.: Hawes. J. E.: Phillips. G. T.: Pryce. R. J.: Ryback. G.: Shears. T. H. J. Chent. Soc. Perkin Trams. I 1994. 1885.

5. For the introduction of nucleophiles at the secondary position of Baylis-Hillman adducts by using the DABCO salt concept. see: (a) Kim. J. N.: Lee. H. J.; Lee, K. Y: Gong, J. H. Symlet 2002. 173. (b) Gong. J. H:: Kim. H. R.: Ryu. E. K:: Kim. J. N. Bull. Korean Chem. Soc. 2002. 23. 789. (c) Kim. J. M.: Lee. K. Y.: Kim. J. N. Bull. Korean Chem. Soc. 2004. 25. 328. (d) Chung. Y. M: Gong. J. H: Kim. T. H.; Kim, J. N. Tetrahedron Lett. 2001, 42 9023. (e) Im. Y. J.; Kim. J. M.: Mun. J. H.: Kim. J. N. Bull. Konam Chem. Soc. $2001,22.349$.

6. Chatterjee. A. K.: Morgat1. J. P.: Scholl. M: Grubbs. R. H. J. Am. Chent. Soc. 2000. 122. 3783 and futher references cited in reference 1

7. (a) Callam. C. S.; Lowary. T. L. J. Ong. Chem. 2001. 66.8961. (b) Camps. P. Colet G: Vazquez. S. ARKIVOC 2003(x). 16

8. (a) Wilt, J. W. Malloy. T. P. Mookerjee, P. K: Sullivan. D. R. J. Org. Chem. 1974. 39. 1327. (b) Wilt. J. W.: Malloy. T. P. J. Am. Chent. Soc. 1970. 92. 4747. (c) Zimmerman. H. E.: Cutshall. T. W. J. Am. Chent Soc 1958. 80. 2893.

9. Lee. J. Y: Kim. J : Lee, K. Y: Kim. J. N. J. Phn Chent A 2004. 108.5678 . 